\title{
THE PARROT UAV CONTROLLED BY PID CONTROLLERS
}

\author{
Andrzej KOSZEWNIK* \\ ${ }^{*}$ Faculty of Mechanical Engineering, Department of Automatic Control and Robotics, Bialystok University of Technology, \\ ul. Wiejska 45C, 15-131 Bialystok, Poland \\ a.koszewnik@pb.edu.pl

\begin{abstract}
The paper presents the process of modeling and designing control laws for four-rotor type of the Parrot UAV. The state space model is obtained by using several phenomena like gyroscopic effects for rigid bodies, propellers and rotors. The obtained model has been used to design PID control laws for roll, pitch, yaw angle and altitude, respectively. The numerical simulations of the closed loop model are shown that system in satisfy way stabilize flight of the quadro-rotor in all considered directions.
\end{abstract}

Key words: PID Control, Parrot, Quadro-Rotor, Modeling

\section{INTRODUCTION}

Autonomous Unmanned Air Vehicle (UAV) are increasingly popular platforms, due to their use in military applications, traffic surveillance, environment exploration, structure inspections, mapping and aerial cinematography. For these applications, the ability of quadro-rotor to take off and land vertically, to perform hover flight as well as their agility, make them ideal vehicles.
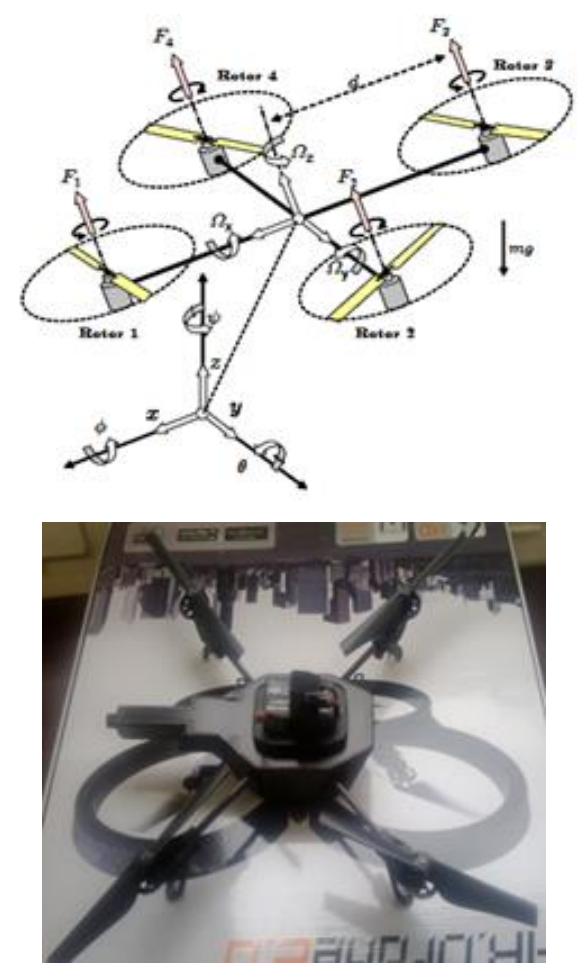

Fig.1. Four-rotor copter and photo of the Parrot

Four rotors helicopters Fig.1 have several basic advantages over manned systems including increased manoeuvrability, low cost, reduced radar signatures. Vertical take-off and landing type
UAV's exhibit further advantages in the manoeuvrability features. Such vehicles require little human intervention from take-off to landing. This helicopter is one of the most complex flying system that exist. This is partly due to the number of physical effects (aerodynamics effects, gravity, gyroscopic, friction and inertial counter torques) acting on the system (Derafa et al., 2007; Kimon, 2007).

Helicopters and quadro-rotors are dynamically unstable and therefore suitable control methods are needed to stabilize them. In order to be able to optimize the operation of the control loop in terms of stability, precision and reaction time, it is essential to know the dynamic behavior of the process which can be established by a representative mathematical model.

\section{DYNAMIC MODELING OF THE PARROT}

The Parrot is equipped with the four-rotor helicopter. Each rotor includes a dedicated brush-less direct current motor, a gearbox and a propeller. The two pairs of propellers $(1,3)$ and $(2,4)$ turn in opposite directions. Forward motion is accomplished by increasing the speed of the rear rotor while simultaneously reducing the forward rotor by the same amount. Left and right motion work in same way. Yaw command is accomplished by accelerating the two clockwise rotating rotors while decelerating the counter-clockwise rotating rotors. Parameters of the Parrot are collected in Tab. 1.

Tab. 1. Parameters of the Parrot (Developer Guide SDK 1.7)

\begin{tabular}{|c|l|l|}
\hline Parameter & Value & Meaning \\
\hline$m$ & $0.38 \mathrm{~kg}$ (outdoor) & Mass (including the support) \\
\hline$l$ & $0.17 \mathrm{~m}$ & $\begin{array}{l}\text { Distance between rotor } \\
\text { and center of mass }\end{array}$ \\
\hline$\Omega$ & $28500 \mathrm{rpm}$ & $\begin{array}{l}\text { Max angular speed } \\
\text { of the rotor -inrunner }-14.5 \mathrm{~V}\end{array}$ \\
\hline$\eta$ & $1 / 8.75$ & $\begin{array}{l}\text { Ratio of angular speed } \\
\text { propeller to rotor }\end{array}$ \\
\hline$\beta$ & $8 \times 3.8$ & Diameter $\mathrm{x}$ stroke of propeller \\
\hline
\end{tabular}


The quadrotor dynamic model is connected with twelve degrees of freedom. The absolute position of the center of mass of quadrorotor is described by $\xi=[x, y, z] T$ and its attitude by the three Euler's angles $\alpha=[\varphi, \theta, \psi] T$. Moreover the model is described by derivatives of absolute position and Eulers's angles $[\dot{x}, \dot{y}, \dot{z}, \dot{\varphi}, \dot{\theta}, \dot{\psi}]^{T}$. These three angles are respectively pitch angle $\left(-\frac{\pi}{2} \leq \phi \leq \frac{\pi}{2}\right)$, roll angle $-\frac{\pi}{2} \leq \theta \leq \frac{\pi}{2}$ and yaw angle $-\pi \leq \psi \leq \pi$.

The dynamic model of the quadrotor describing the roll, pitch and yaw rotations contains three terms which are: the gyroscopic effect resulting from the rigid body rotation, the gyroscopic effect resulting from the propeller rotation coupled with the body rotation and finally the action of the actuators:

$I_{X X} \ddot{\phi}=\dot{\theta} \dot{\psi}\left(I_{y y}-I_{z z}\right)+J_{r} \dot{\theta} \Omega_{r}+l\left(-\Omega_{2}{ }^{2}+\Omega_{4}{ }^{2}\right)$

$I_{Y Y} \ddot{\phi}=\dot{\phi} \dot{\psi}\left(I_{z Z}-I_{x x}\right)-J_{r} \dot{\theta} \Omega_{r}+l\left(-\Omega_{1}{ }^{2}+\Omega_{3}{ }^{2}\right)$

$I_{z z} \ddot{\psi}=\dot{\phi} \dot{\theta}\left(I_{x x}-I_{y y}\right)+J_{r} \dot{\Omega}_{\mathrm{r}}$

where: $I_{X X}$ - moment of interia about $\mathrm{X}$ axis, $I_{Y Y}$ - moment of interia about $Y$ axis, $I_{Z Z}$ - moment of interia about $Z$ axis, $\Omega_{1}, \Omega_{2}, \Omega_{3}, \Omega_{4}$ - angular speeds of the rotors, $\Omega_{r}$ - angular speed of the propeller

Moreover the quadrorotor model describes the position of the Parrot versus the horizon. Neglecting hub forces and friction action, the equations in $x, y, z$ directions are determined via the Newton-Euler formalism:

$m \ddot{z}=m g-(\cos \psi-\cos \phi) \sum_{i=1}^{4} \Omega_{i}^{2}$

$m \ddot{x}=(\cos \phi \sin \theta \cos \psi+\sin \phi \sin \psi) \sum_{i=1}^{4} \Omega_{i}{ }^{2}$

$m \ddot{y}=(\cos \phi \sin \theta \sin \psi-\sin \phi \cos \psi) \sum_{i=1}^{4} \Omega_{i}{ }^{2}$

In the next step, the model of the Parrot described by $\mathrm{Eq}(1)$ and $\mathrm{Eq}(2)$ is simplified, by inserting new constant values (a1,...a5 and $b 1 . . b 3$ ), input signals $\mathrm{U} 1, \ldots \mathrm{U} 4$ and parameters $u x$ and $u y$. Then the model of the quadro-rotor is transformed to(Clavel et al., 2007):

$$
\dot{X}=f(X, U)=\left(\begin{array}{c}
\dot{\phi} \\
\dot{\theta} \dot{\psi} a_{1}+\dot{\theta} a_{2} \Omega_{r}+b_{1} U_{2} \\
\dot{\theta} \\
\dot{\phi} \dot{\psi} a_{3}+\dot{\phi} a_{4} \Omega_{r}+b_{2} U_{3} \\
\dot{\psi} \\
\dot{\theta} \dot{\phi} a_{5}+b_{3} U_{4} \\
\dot{z} \\
-g+(\cos \phi \cos \theta) \frac{1}{m} U_{1} \\
\dot{x} \\
u_{x} \frac{1}{m} U_{1} \\
\dot{y} \\
u_{y} \frac{1}{m} U_{1}
\end{array}\right)
$$

with

$$
\begin{cases}a_{1}=\left(I_{Y Y}-I_{Z Z}\right) / I_{X X} \mid & a_{5}=\left(I_{X X}-I_{Y Y}\right) / I_{Z Z} \mid \\ a_{2}=-J_{r} / I_{X X} \mid & b_{1}=l / I_{X X} \mid \\ a_{3}=\left(I_{Z Z}-I_{X X}\right) / I_{Y Y} \mid & b_{2}=l / I_{Y Y} \mid \\ a_{4}=J_{r} / I_{Y Y} \mid & b_{3}=1 / I_{Z Z} \mid\end{cases}
$$

and

$u_{x}=(\cos \phi \sin \theta \cos \psi+\sin \phi \sin \psi)$

$u_{y}=(\cos \phi \sin \theta \sin \psi-\sin \phi \cos \psi)$

and

$\left\{\begin{array}{l}U_{1}=b\left(\Omega_{1}{ }^{2}+\Omega_{2}{ }^{2}+\Omega_{3}{ }^{2}+\Omega_{4}{ }^{2}\right) \\ U_{2}=b\left(-\Omega_{2}{ }^{2}+\Omega_{4}{ }^{2}\right) \\ U_{3}=b\left(\Omega_{1}{ }^{2}-\Omega_{3}{ }^{2}\right) \\ U_{4}=d\left(-\Omega_{1}{ }^{2}+\Omega_{2}{ }^{2}-\Omega_{3}{ }^{2}+\Omega_{4}{ }^{2}\right)\end{array}\right.$

where: $b$ - thrust coefficent of rotor, $d$ - drag coefficent of rotor.

As we can see in $\mathrm{Eq}(3)$ the model of the quadrotor is rewritten in the state space form $\dot{X}=f(x, u)$ with the inputs vector $u$ and the state vector $x$ chosen as follows:

$x=\left[\begin{array}{llllllllllll}\phi & \dot{\phi} & \theta & \dot{\theta} & \psi & \dot{\psi} & z & \dot{z} & x & \dot{x} & y & \dot{y}\end{array}\right]^{T}$

$u=\left[\begin{array}{llll}U_{1} & U_{2} & U_{3} & U_{4}\end{array}\right]^{T}$

Then the obtained model in the state space form is expressed by:

$\dot{\mathbf{X}}=\mathbf{A} \boldsymbol{x}+\mathbf{B} \boldsymbol{u}$
$y=\mathbf{C} \boldsymbol{x}+\mathbf{D u}$

where:

$\mathbf{A}_{12 \times 12}=\left[\begin{array}{cccccccccccc}0 & 1 & 0 & 0 & 0 & 0 & 0 & 0 & 0 & 0 & 0 & 0 \\ 0 & 0 & 0 & a_{24} & 0 & 0 & 0 & 0 & 0 & 0 & 0 & 0 \\ 0 & a_{32} & 0 & 1 & 0 & 0 & 0 & 0 & 0 & 0 & 0 & 0 \\ 0 & 0 & 0 & 0 & 0 & 0 & 0 & 0 & 0 & 0 & 0 & 0 \\ 0 & 0 & 0 & 0 & 0 & 1 & 0 & 0 & 0 & 0 & 0 & 0 \\ 0 & 0 & 0 & 0 & 0 & 0 & 0 & 0 & 0 & 0 & 0 & 0 \\ 0 & 0 & 0 & 0 & 0 & 0 & 0 & 1 & 0 & 0 & 0 & 0 \\ 0 & 0 & 0 & 0 & 0 & 0 & 0 & 0 & 0 & 0 & 0 & 0 \\ 0 & 0 & 0 & 0 & 0 & 0 & 0 & 0 & 0 & 1 & 0 & 0 \\ 0 & 0 & 0 & 0 & 0 & 0 & 0 & 0 & 0 & 0 & 0 & 0 \\ 0 & 0 & 0 & 0 & 0 & 0 & 0 & 0 & 0 & 0 & 0 & 1 \\ 0 & 0 & 0 & 0 & 0 & 0 & 0 & 0 & 0 & 0 & 0 & 0\end{array}\right]$

where:

$a_{32}=\psi \cdot a_{3}+a_{4} \cdot \Omega_{\mathrm{r}}$.

$a_{24}=\psi \cdot a_{1}+a_{2} \cdot \Omega_{\mathrm{r}}$

$\mathbf{B}_{12 \times 4}=\left[\begin{array}{cccc}0 & 0 & 0 & 0 \\ 0 & b_{1} & 0 & 0 \\ 0 & 0 & 0 & 0 \\ 0 & 0 & b_{2} & 0 \\ 0 & 0 & 0 & 0 \\ 0 & 0 & 0 & b_{3} \\ 0 & 0 & 0 & 0 \\ (\cos \phi \cos \theta) / m & 0 & 0 & 0 \\ 0 & 0 & 0 & 0 \\ u_{x} / m & 0 & 0 & 0 \\ 0 & 0 & 0 & 0 \\ u_{y} / m & 0 & 0 & 0\end{array}\right] ;$ 


$$
\left.\mathbf{C}_{12 \times 12}=\left[\left[\begin{array}{ccc}
1 & \cdots & 0 \\
\vdots & \ddots & \vdots \\
0 & \cdots & 1
\end{array}\right]\right] ; \quad \mathbf{D}_{12 \times 4}=\left[\begin{array}{ccc}
0 & \cdots & 0 \\
\vdots & \ddots & \vdots \\
0 & \cdots & 0
\end{array}\right]\right]
$$

The dynamic model of the open loop system is expressed by matrices which values are derived from calculations and assumptions. Some of these parameters are calculated on basic parameters given in Tab.1 and Tab. 2 .

Tab. 2. The parameters calculated and assumed for simulation (Parrot Ar Drone)

\begin{tabular}{|l|l|l|}
\hline Parameter & Values & Information \\
\hline$J_{x x}$ & $0.0086[\mathrm{kgm} 2]$ & Calculated \\
\hline$J_{y y}$ & $0.0086[\mathrm{kgm} 2]$ & Calculated \\
\hline$J_{z z}$ & $0.0172[\mathrm{kgm} 2]$ & Calculated \\
\hline$b$ & $3.13 \mathrm{e}-5$ & Assumed \\
\hline$d$ & $7.5 \mathrm{e}-7$ & Assumed \\
\hline $\mathrm{Jr}$ & $6 \mathrm{e}-5[\mathrm{kgm} 2]$ & Assumed \\
\hline
\end{tabular}

\section{DESIGN OF THE CONTROL LAW}

The main objective is to design the classic control law in order to stabilize the yaw, pitch and roll angles and the altitude of the
Parrot. Stabiliziation of the platform is achived by designing four separate PID controlers controling pitch, yaw roll angles and the altitude position, respectively. Since the input signals of the model are angular velocities of particular rotors, so the obtained values of the control signals in the closed loop system need to be multiplied by the gain matrix $\mathrm{K}$, represents transformation controller outputs to speed inputs for each rotor.The obtained block diagram with all controllers is shown in Fig. 3.

$\mathbf{\Omega}_{\mathbf{i}}=U_{i} * \mathbf{K}$

where: $i$ - $i$-th rotor of the Parrot $(i=1, . ., 4)$

$\mathbf{K}=\left[\begin{array}{cccc}\frac{1}{4 b} & \frac{1}{4 b} & \frac{1}{4 b} & \frac{1}{4 b} \\ 0 & -\frac{1}{2 b l} & 0 & \frac{1}{2 b l} \\ \frac{1}{2 b l} & 0 & -\frac{1}{2 b l} & 0 \\ -\frac{1}{4 d} & \frac{1}{4 d} & -\frac{1}{4 d} & \frac{1}{4 d}\end{array}\right]=$

$1 e 5 \cdot\left[\begin{array}{cccc}0.0799 & 0.0799 & 0.0799 & 0.0799 \\ 0 & -0.9397 & 0 & 0.9397 \\ 0.9397 & 0 & -0.9397 & 0 \\ -3.333 & 3.333 & -3.333 & 3.333\end{array}\right]$

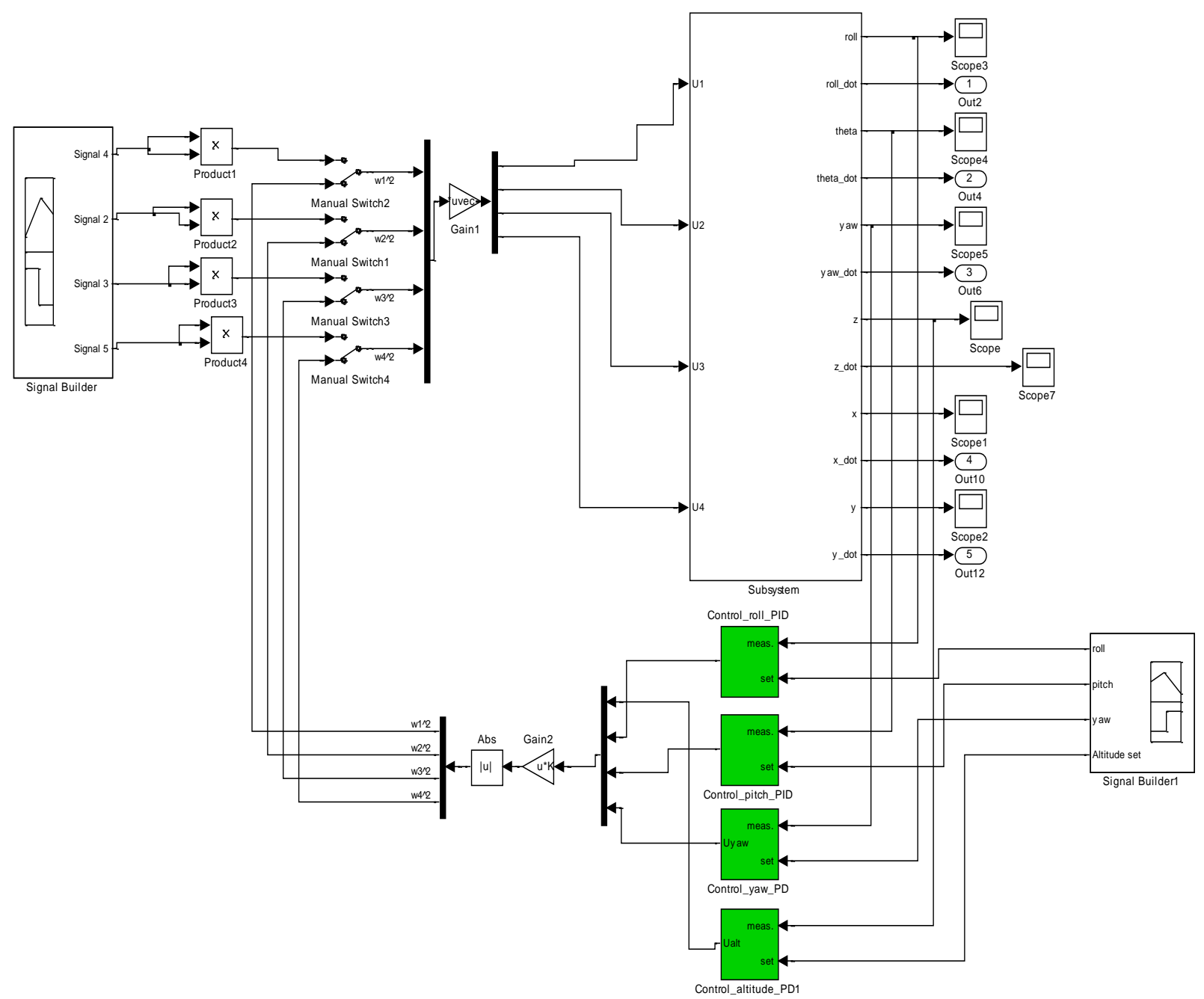

Fig. 2. Block diagram of the closed-loop system 
In the first step the altitude controller is designed to stabilize the vertical position of the platform. The first input of the controller is the altitude error that is the difference between the altitude value and the measurement value and the second - velocity in $Z$ direction $-\dot{z}$ as altitude_d_error. The control law of this PID controller is as follows (Bouabdalth and North, 2004):

$\dot{e}_{z}=\dot{z}$

$e_{z}=z_{S E T}-z$

$u_{z}=k_{p} \cdot e_{z}+k_{i} \cdot e_{z}-k_{d} \cdot \dot{e}_{z}$

where: $Z_{-}$dot - velocity of the Parrot in $Z$ direction, $Z_{S E T}$ - desired altitude of the Parrot in $Z$ direction.

Finally, the transfer function of the controller is:

$P I D(s)=k_{d} s+k_{i} / s+k_{p}$

During the simulations the changes of pitch, roll and yaw angles are assumed as zero. As a result, the PID controller generated only control signal U1 that caused hovering or falling of the model in vertical direction. Ipso facto all lift forces generated by rotors have the same value. Parameters of such PID controller are chosen by using the trial and error method and equal to: $k_{p \_}$alt $=12, k_{\text {i_alt }}=1.5, k_{d}$ alt $=6$, respectively.

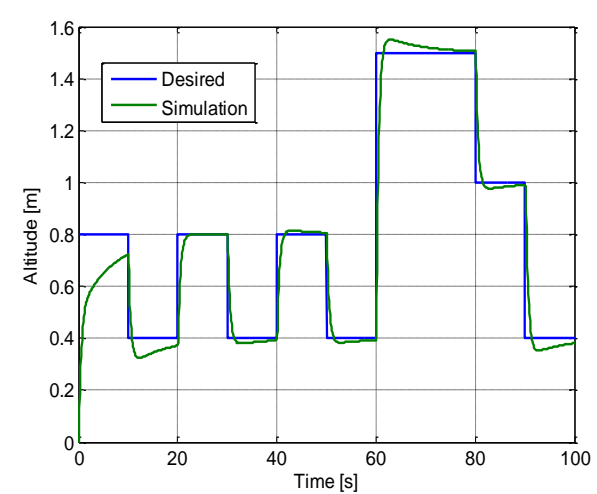

Fig. 3. Altitude response of the closed-loop system

As we can see in Fig.3, the closed loop system is characterized by very short time constant, specially in time range $20-100 \mathrm{~s}$. Taking into account the first 20 seconds of simulation we can see that the system does not achive desired value of $0.8 \mathrm{~m}$. Perhaps it was caused by earlier assumption that the initial conditions of simulation equal to zero.

The next step of the simulation is connected with the design of the pitch controller. Once again, based on two signals: pitch angle and velocity change of pitch angle - theta_dot the parameters of the controller are chosen as follows: $k_{p}$ teta $=0,2$, $k_{i}$ teta $=0 k_{\text {d_t }}$ teta $=0,15$, respectively. Finally the transfer function of the controller is:

PID_teta $(s)=0.15 s+0.2$

The controller PID_teta $(s)$ generates the control signal U3 directly influencing the angular velocity of both front and rear rotors and indirectly influencing control signals U1 and U4. Therefore, in order to verify the parameters of the pitch controller the desired trajectory of pitch and roll angles and the altitude should be included.

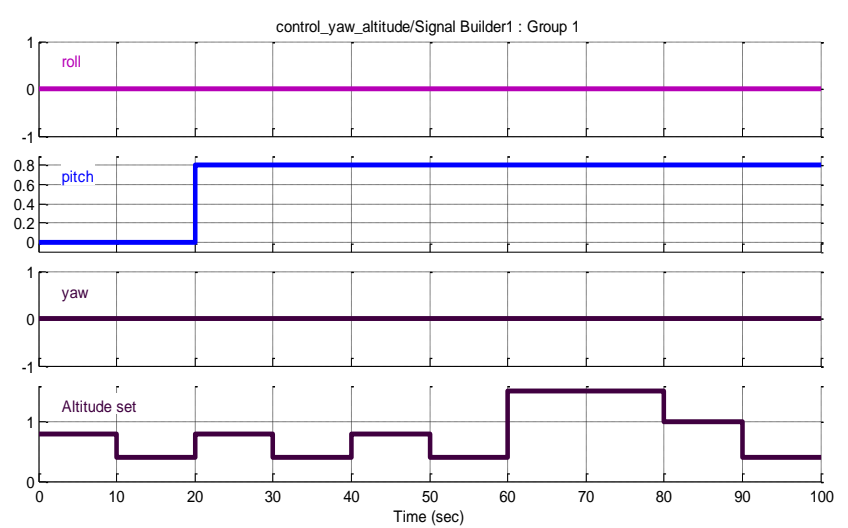

Fig. 4. Desired trajectory of the pitch angle and the altitude
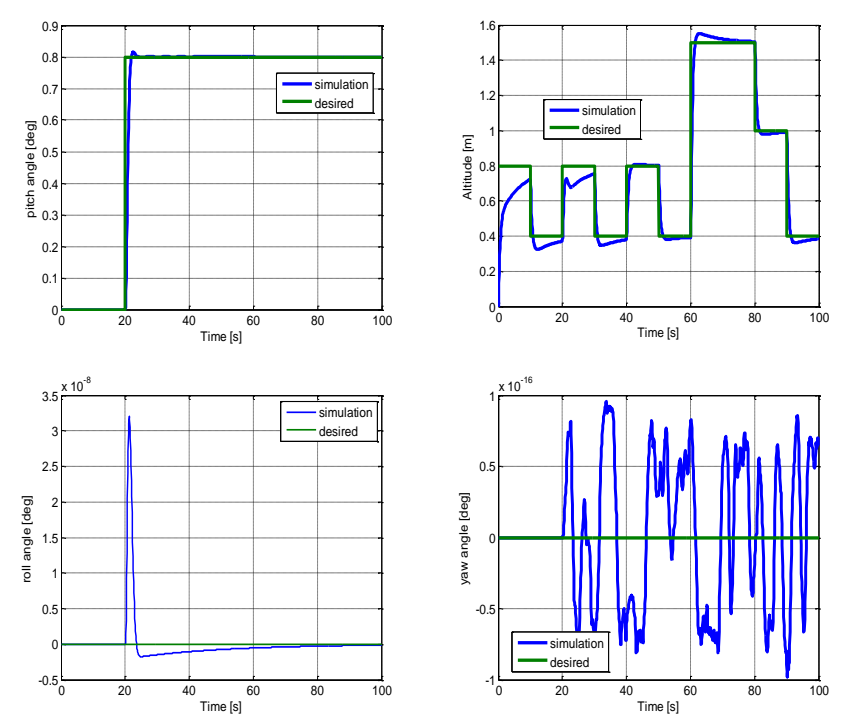

Fig. 5. Response of the closed loop system to the desired trajectory of the pitch angle and the altitude

According to Fig.5 the parameters of the pitch controller are correct. Measurement value of the pitch angle follows the desired trajectory. Moreover both pitch and altitude signals are coupled what is shown on apropriate plots. The small overshoot of the pitch angle at the 22 second of simulation caused small falling of the model. After that both signals once again tried achive the set value. At this same times others signals: roll and yaw angle still have values of zero. Such bahaviour of the closed loop model of course it is a correct.

The next stage of simulation is connected with the design of the yaw controller. In order to choose proper values the parameters of the controller yaw and yaw_dot signals are taken as inputs. Finally, the parameters of the controller are as follow:

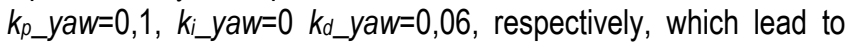
the transfer function described as:

PID_yaw $(s)=0.06 s+0.1$

The yaw controller generated the control signal U4 which influenced only the yaw angle. Thus, change of yaw angle does not caused change of other angles and altitude of the model. Moreover, how we can see in Fig.7 the model with PID_yaw controller very fast achived desired value. So, the closed loop model according with desired impulse very fast in first step rotates in right side and next left side. 


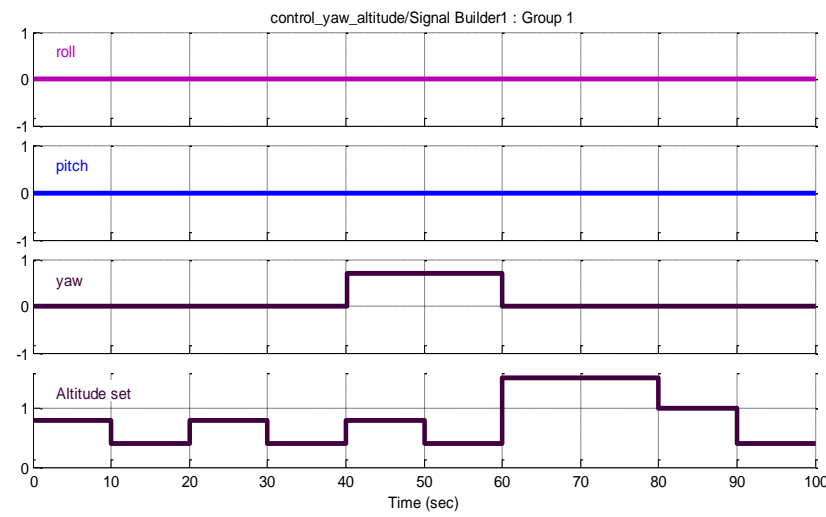

Fig. 6. Desired trajectory of the yaw angle and the altitude
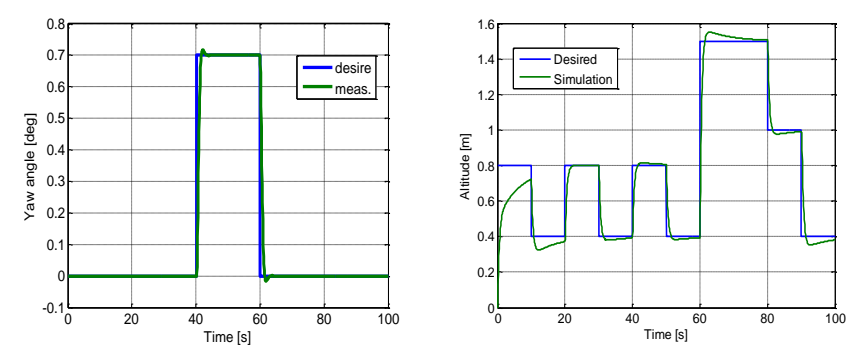

Fig. 7. The closed loop system response wih PID_yaw and PID_altitude controlles

The last controller is the roll controller. Once again and similar to previous cases, the roll and roll_dot signals are chosen as input signals. Based on the simulations the parameters of the controller are chosen and equal: $k_{p} \_$yaw $=0.3, k_{i}$ yaw $=0.01 k_{d} \_$yaw $=0.25$, respectively.
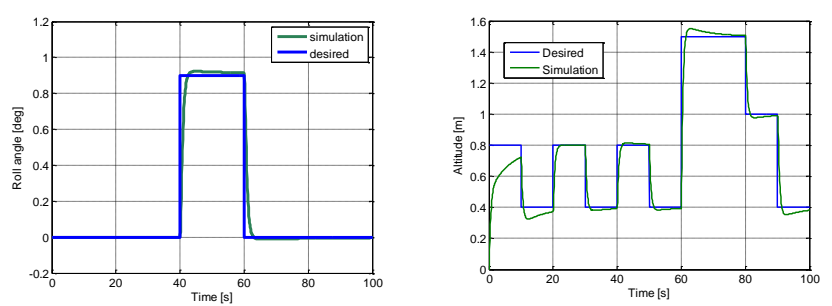

Fig. 8. The closed loop system response with PID_roll and PID_altitude controllers

As we can see in Fig.8 also in this case the controller immediately stabilizes the roll angle. Especially, it is shown in the roll angle plot where the rising and falling times are very short. Furthermore, according to Fig. 8 the change of the angular velocity of all rotors casued by the change of the control signal U2 does not infulence the altitude of the model. Such situation is also correct, because stablilization of the altitiude is achieved by the independent altitude controller.

\section{SUMMARY AND CONCLUSIONS}

The paper shows the modelling and control of the quadro-rotor of the Parrot. The first part is connected with derivation of the mathematical model by using Newton-Euler method. The obtained model is a MIMO model, so in the next step the model is simpilified and rewritten to the state space form with 12 variables of the state space vector.

Then the model is decoupled and for each signals: roll, pitch and yaw angles and the altitude the PID controllers are designed. The obtained results for all controllers proved that the whole closed loop system correctly stabilizes the motion of the Parrot.

In further investigations, the design of the global control law beetwen two Parrots will be considered. One of them will be a leader and the second will be a follower. Then, the results of such simulations for both Parrots (Leader-Follower) will be implementated to the auto-pilot in order to verify the control algorithms.

\section{REFERENCES}

1. Ambroziak L., Gosiewski Z., Ołdziej D. (2012), Modeling four rotors Helicopter, Chapter in monogrpaphy: Elements of autonomous flight of UAV, Białystok University of Technology (in Polish).

2. Astrom K. (2002), Control System Design, Chapter PID control, http://www.cds.caltech.edu/ murray/courses/cds101/fa02/caltech/ast rom-ch6.pdf (20.02.2014).

3. Bouabdalth S., Noth A. (2004), PID vs LQ Control Techniques Applied to Indoor Micro Quadrotor, IROS.

4. Clavel R. Siegwert R, Corke P. (2007), Design and Control Quadrotors with Application to Autonomous Flying, Monography with Ecole Politechnique Federale de Laussane.

5. De Silva D. (2012), Formation Control for Unmanned Aerial Vehicles, https://fenix.tecnico.ulisboa.pt/downloadFile/395144217739/Resumo Alargado.pdf (20.02.2014).

6. Derafa L., Madani T., Benalleque A. (2006), Dynamic Modeling and Experimental Identification of Four Rotors Helicopter Parameters, IEEE International Conference on Industrial Technology, pp.265-272.

7. Derafa L., Madani T., Ouladi A., Benallegue A. (2007), Four Rotors Helicopter Yaw Altitude Stabilization, Proceedings of the World Congress on Engineering, Vol. I, pp.104-110

8. Kimon P. (2007), Advances in Unmanned Aerial Vehicle. State of the Art and Road to Autonomy, Publisher: Springer

9. McKerrow P. (2004), Modeling the Dragonflyer Four-Rotor Helicopter, International Conference on Robotics \& Automation, pp.35963601.

10. Parrot Ar Drone, Developer Guide SDK 1.7. 